\title{
Etude comparative des formes de discours rapportés dans des contes des origines chez des scripteurs français et brésiliens (7-8 ans) : premiers résultats
}

\author{
Catherine Boré ${ }^{\text {, Eduardo Calil de Oliveira }}{ }^{2}$, et Kariny Louiza Amorim² \\ ${ }^{1}$ Université de Cergy-Pontoise, Laboratoire EMA EA 450, France \\ ${ }^{2}$ Université Fédérale d'Alagoas, Laboratoire LAME, Macéio, Brésil
}

\begin{abstract}
Résumé. Le discours rapporté joue un rôle essentiel dans les récits de fiction, attestant de la capacité du scripteur à présenter le point de vue d'autrui, mais il reste peu étudié en acquisition de l'écriture. Cet article présente les premiers résultats d'une enquête didactique réalisée en contraste de langue chez des élèves de 7-8 ans récemment alphabétisés, issus d'une école française et d'une école brésilienne, et placés dans des conditions de production identiques. Les chercheurs se situent dans une perspective interactionniste, socioconstructiviste, appuyée sur la psychologie culturelle. La présentation analyse trois séries de productions recueillies respectivement dans chaque école: deux productions de contes des origines à sujet libre, et une troisième à sujet imposé impliquant l'emploi de discours rapporté. Au total 57 textes écrits par dyades sont analysés, 20 brésiliens et 37 français.

Les résultats montrent que, sur les trois productions, les élèves brésiliens écrivent des textes plus longs et qui comportent plus de discours rapporté que les élèves français, mais que l'écart en nombre de mots se resserre quand on n'envisage que les textes comportant du discours rapporté. Significativement, la troisième production à thème imposé marque une augmentation des discours rapportés dans les deux pays avec une similitude dans les constructions. Plus généralement, le lexique des verbes introducteurs est identique pour la moitié de ses occurrences. Ces résultats pointent de nombreuses questions liées aux priorités d'enseignement notamment de la grammaire dans la culture didactique des deux pays.
\end{abstract}

\section{Introduction}

Nous présentons le début d'une étude comparative concernant un aspect particulier de la production d'écrits de jeunes scripteurs récemment entrés en littératie, le discours rapporté, en précisant plus loin l'étendue que nous donnerons à cette notion complexe.

Notre étude est d'abord motivée par le très petit nombre de recherches ayant trait à cette question à l'écrit, alors qu'il existe pour l'oral des monographies ou des synthèses importantes sur l'acquisition du langage incluant le discours rapporté [1] Bonnet \& Tamine, 1984, [2] Lentin et al. 1984, 1988, [3] Lentin, 2009, [4] Bernicot, Veneziano et al. 2010, [5] (Goodell et Sachs, 1992) pour le domaine anglo-saxon. 
Une revue rapide des études consacrées au discours rapporté visant spécifiquement l'acquisition des jeunes apprenants à l'écrit montre qu'elles sont peu nombreuses ; d'abord parce que la plupart des études concernant l'apprentissage de l'écrit chez le jeune scripteur sont généralistes [6] Simon, 1973, [7] Schneuwly 1988, [8, 9] Fayol $(1985,1997)$ parmi une foule de références du domaine français, et n'ont pas pour objet principal le discours rapporté.

D'autre part, celles qui existent sont souvent anciennes : [10] de Gaulmyn, 1983, [11] Foulin \& Fayol, 1988 ; la plupart se situe dans la période 1980-1995 : [12] Hickmann, (1991), [13] (Dittmar \& Reich, 1993).

En Amérique Latine, les études concernant l'acquisition du langage écrit en portugais en contraste de langues sont rares. On rappelle la grande étude présentée dans l'ouvrage Chapeuzinho Vermelho aprende a escrever : estudos psicolinguísticos comparativos em três línguas (1996). Dans ce travail collectif, publié il y a près de 20 ans, les chercheures [14] Ferreiro, Pontecorvo, Moreira et Garcia Hidalgo étudient Le Petit Chaperon Rouge, réécrit par des élèves locuteurs du portugais, de l'espagnol et de l'italien dans des écoles de l'enseignement élémentaire au Brésil, au Mexique, en Uruguay et en Italie. Mais cette étude concerne surtout le rapport entre discours direct et ponctuation et s'appuie sur une tâche de réécriture plutôt que d'invention. On peut citer encore les études de [15] Figueiredo (1998) et [16] Soares (1999).

En France, c'est finalement en didactique du français que l'on trouve le plus de recensions : citons le travail de [17] Granget (2008) en didactique du français langue étrangère mais sur une tranche d'âge différente avec des locuteurs allemands ; ou, surtout, en didactique du français langue maternelle : [18] Fabre (1992) ; [19-22] Boré, (2004 (a et b), 2009, 2010) ; [23] Plane et al., ( à paraître 2013).

Si bien que notre étude est probablement à notre connaissance une des plus récentes réunissant à la fois trois caractéristiques :

- Traiter du discours rapporté à l'écrit et non à l'oral ;

- Concerner de jeunes scripteurs tout juste entrés en littératie ;

- Constituer une étude contrastive franco-brésilienne en didactique.

Nous nous situons en didactique en effet : nous pensons que le cadre et le dispositif didactiques peuvent favoriser la maîtrise de l'écrit plus que le développement psychologique interne des jeunes scripteurs. Le cadre théorique concernant la perspective d'acquisition est celui du socio- constructivisme [24] Vygotski, 1934, [25] Bronckart, 1997 et de la psychologie culturelle [26] Bruner, 2008 : nous considérons que le développement ne peut se produire que par les médiations sociales notamment entre pairs ; c'est pourquoi les dispositifs didactiques ont été construits pour permettre une écriture collaborative en dyades constamment étayée par le dialogue et par les œuvres culturelles présentes ou recréées par la mémoire discursive.

Dans cette perspective, le discours rapporté n'est pas un objet détaché du récit qui serait susceptible d'un apprentissage grammatical autonome ; il manifeste l'aptitude du jeune scripteur à l'altérité par la reprise des mots de l'autre.

Nous considérons en effet comme centrale la représentation de la parole dans l'activité humaine. Il s'agit là d'un fait inséparablement linguistique, langagier et anthropologique développé par Bakhtine et Volochinov, puis dans le cadre de la psychologie culturelle (Bruner) et pris en compte dans les théories de l'imagination qu'elles soient culturelles [27] P. Harris [2000] (2007) ou cognitives [28] Reboul (2000). La centralité de la parole représentée dans la vie du langage alimente l'hypothèse qui a sous-tendu cette étude : s'il est vrai que le discours rapporté joue un rôle nécessaire dans le développement de l'écriture des fictions narratives, la variété et le nombre des dialogues et discours représentés pourraient témoigner de la capacité des scripteurs à inventer des histoires. 


\section{Cadre théorique : dialogisme et Discours Rapporté (DR)}

Nous situons notre étude des discours rapportés dans le cadre du dialogisme bakhtinien. C'est dans la section « Du discours romanesque » d'Esthétique et théorie du roman ${ }^{1}$ de [29] Bakhtine que nous trouvons certains développements consacrés au concept de dialogisme. Aucune définition n'est donnée par Bakhtine mais la lecture de cette célèbre section a donné lieu à deux importantes interprétations françaises, celle de [30] J. Authier-Revuz (1995) et celle de [31] Brès \& Nowakowsjka (2006) qui distinguent trois types de dialogisme à partir des distinctions que fait Bakhtine lui-même. S'il est impossible de résumer le foisonnement et la complexité de ce chapitre, nous retenons certains passages comme points de départ de nos travaux.

« Le dialogue a été étudié seulement comme forme compositionnelle de la structure de la parole. Mais la dialogisation intérieure (tant dans la réplique que dans l'énoncé monologique) qui pénètre dans toute sa structure, dans toutes ses couches sémantiques et expressives, a presque toujours été ignorée. $[\ldots]$

Le discours naît dans le dialogue comme sa vivante réplique et se forme dans une action dialogique mutuelle avec le mot d'autrui, à l'intérieur de l'objet. ${ }^{2}$ Le discours conceptualise son objet grâce au dialogue. $»^{3}$

C'est donc cette action dialogique avec le mot d'autrui qui sert d'entrée à notre conception du discours rapporté. Nous retenons les caractéristiques fondamentales dégagées par Bakhtine dans ce même chapitre :

« [...] Il suffit d'écouter et de méditer les paroles qu'on entend partout pour affirmer ceci : dans le parler vivant de tout homme vivant en société, la moitié au moins des paroles qu'il prononce sont celles d'autrui (reconnues comme telles) transmises à tous les degrés de possibles d'exactitude et d'impartialité (ou plutôt de partialité).

[...] Il est indispensable de noter ceci : la parole d'autrui comprise dans un contexte, si exactement transmise soit-elle, subit toujours certaines modifications de sens. Le contexte qui englobe la parole d'autrui crée un fond dialogique dont l'influence peut être fort importante. [...] La parole d'autrui, introduite dans le contexte d'un discours, établit avec le contexte qui l'enchâsse non pas un contact mécanique, mais un amalgame chimique (au plan du sens et de l'expression); le degré d'influence mutuelle par le dialogue peut être très grand. Voilà pourquoi, lorsqu'on étudie les différentes formes de transmission du discours d'autrui, on ne peut séparer le procédé d'élaboration de ce discours du procédé de son encadrement contextuel (dialogique) : les deux procédés sont indissolublement liés. » ${ }^{4}$

Le discours rapporté, qu'il soit représenté dans le langage ordinaire ou dans les textes littéraires, est donc expressément mentionné par Bakhtine comme un fait de dialogisme, en raison du contexte qui unit et sépare à la fois les deux énonciations. C'est en effet dans un seul énoncé que se trouvent associées deux énonciations émanant d'énonciateurs distincts. Le mode d'association, cependant, est variable : dans le discours direct, les énonciations sont juxtaposées et le contraste des situations d'énonciation se marque par des modifications affectant grammaticalement les temps, les modalités et les personnes. Mais les éléments de représentation du mot d'autrui au discours direct peuvent s'accompagner d'une coloration des contextes dans lesquels apparait l'énoncé étranger : par exemple, l'utilisation d'un lexique spécifique différent dans les deux énonciations. Un exemple saisissant nous est fourni par les onomatopées que les jeunes scripteurs présentent au discours direct dans les textes qu'ils inventent [32] Calil \& Del Ré (2009), [33] Calil \& Boré (2011). Ce discours « étranger » est intégré par les scripteurs sans verbe

\footnotetext{
${ }^{1}$ Bakhtine M., ([1934, 1975]1978), pp. 83-233.

2 Les italiques sont ajoutées.

3 Bakhtine M., ibidem, pp. 102-103.

${ }^{4}$ Ibidem, pp. 158-159.
} 
introducteur, dans une collision violente avec les mots du narrateur, mais nous décelons bien qu'il s'agit de mots empruntés aux « comics » populaires ou aux contes pour enfants

Un autre mode d'association est celui du discours indirect, qui prend le plus souvent une forme syntaxique de subordination ${ }^{5}$ mais dont la variété des formes est très étendue : en fait, le discours indirect est le lieu d'une réappropriation des mots d'autrui par l'énonciateur et corrélativement, comme Bakhtine le signale, d'une déformation/assimilation de ce « discours autre » (Authier-Revuz) par l'énonciateur principal, qui transmute dans son propre langage les mots d'autrui, en laissant parfois au passage des expressions, guillemetées ou non, appartenant au discours rapporté ${ }^{6}$. Cette façon de faire cohabiter deux langages en un seul est moins visible que le discours direct, mais elle illustre une forme d'hétérogénéité « constitutive » (Authier-Revuz) qui peut se prolonger dans des ramifications innombrables : par exemple, une variété du discours indirect est le discours « narrativisé », qui mentionne seulement le fait qu'un ou plusieurs énonciateurs parlent, et donc ne rapporte qu'un acte de langage ; mais il y a toute une gamme possible de représentations de cet acte de langage, par exemple : "ils ont discuté de leurs vacances pendant des heures »; "ils se sont amusés à raconter leurs récits de vacances »; ils se sont émus en évoquant leurs souvenirs de vacances »; "ils ont échangé des confidences à propos de leurs vacances » etc. Une infinie variété de perspectives est ouverte par l'énonciateur dont la subjectivité est ici interprétante du ton, de l'atmosphère et du contexte portés par l'événement de langage rapporté.

Enfin, la représentation la plus célèbre du dialogisme incarné dans le discours rapporté se trouve dans ce que Bakhtine nomme «bivocalité », que nous connaissons sous la forme du « discours indirect libre » : dans les analyses qui suivent, nous verrons que les jeunes scripteurs y recourent, d'une manière parfois difficile à discerner, chaque fois que la voix de l'énonciateur principal et celle de l'énonciateur rapporté se confondent, se superposant de telle sorte qu'on ne peut pas les attribuer de façon certaine au narrateur ou au locuteur représenté : « il était une fois une femme qui voulait un enfant » : est-ce le propos de la femme qui dit: « je veux un enfant » qui est rapporté, ou bien est-ce l'énoncé du narrateur qui décrit le désir de la femme ? Les deux voix se fondent dans la même énonciation, marquée par l'interprétation qu'en fera le lecteur : l'ambivalence manifeste ici la fusion de deux voix dans un même énoncé, manifestation majeure d'un dialogisme à l'œuvre dans la langue et sans doute non concerté de la part du scripteur.

Le discours indirect libre avait été analysé par [34] Volochinov dès 1929 dans sa grande étude sur les discours rapportés ${ }^{7}$. Pour lui, ce dialogisme est de nature sociale et non psychologique ou expressive :

«Ce n'est pas le Mot qui est l'expression de la personne intérieure, mais c'est la personne intérieure qui est le Mot exprimé ou intériorisé. Le Mot est l'expression de l'échange social, de l'interaction sociale des personnes matérielles, des producteurs. ${ }^{8}$

Cette conception est assez proche des analyses du psychologue [24] Lev Vygotski concernant le langage intérieur et sa socialisation. Boré dans ses travaux récents [21, 22] $(2009,2010)$ a repris la thèse vygostkienne selon laquelle l'activité de l'enfant est modelée sur et par ses rapports avec autrui par l'intermédiaire du langage, elle rappelle que c'est dans le dialogue socialisé, externe, en face à face, que l'enfant commence ses premiers apprentissages symboliques, dialogue qui s'intériorise progressivement. Boré a développé l'hypothèse que les dialogues représentés dans les récits de fiction sont des traces fonctionnelles de ce langage intérieur, qui présente des analogies avec le dialogisme

\footnotetext{
5 En français, principalement avec la subordination par « que ». Exemple: « Il a dit que etc. » mais aussi par l'infinitif. Exemple : «Il lui a demandé de se taire ».

6 J. Authier-Revuz nomme cette forme « îlots textuels ». Exemple : «Il a dit qu'il viendrait « sûrement » ».

7 V.N. Voloshinov ([1929, 1930], 2010). Le marxisme et la philosophie du langage, traduction de Patrick Sériot et Inna TylkowskiAgeeva, édition bilingue, chapitres III et IV, Limoges: Lambert-Lucas, pp.385-466.

C'est dans ces chapitres que l'on trouve les analyses les plus détaillées concernant «l'orientation réciproque de la parole d'auteur et de la parole d'autrui », en particulier le chapitre IV pour le discours indirect libre.

8 Volochinov, op.cit. chapitre IV p. 451.
} 
bakhtinien. Ainsi se trouve posée l'hypothèse du statut du dialogue dans le processus d'invention comme trace d'un dialogisme manifesté. C'est ce qui permet à Boré de dire que le dialogue est « le moteur de l'invention » [21] Boré (2009).

\section{Dispositif didactique et observations générales}

Nous considérons ce travail comme un « pilote », un test, nous permettant d'expérimenter dans un $1^{\text {er }}$ temps tous les aspects complexes avant d'en corriger les défaillances et de l'étendre à d'autres écoles.

C'est aussi une recherche empirique, faite dans des conditions écologiques, quantifiée plutôt que quantitative, avec une part importante d'analyses qualitatives.

Voyons maintenant le dispositif.

Le corpus des manuscrits brésiliens et français a été recueilli dans deux classes, une brésilienne ${ }^{9}$ et une française ${ }^{10}$ auprès d'élèves de même âge (7-8 ans) issus de milieux socio-économiques approchants, et ayant accès à la culture écrite véhiculée par la littérature pour enfants, les films, les émissions de télévision, les jeux électroniques, etc.

Les textes ont été écrits entre mars et juillet 2011 à partir de situations de production de textes similaires.

L'équipe pédagogique de chaque école partage en effet une même conception de l'enseignement de la langue maternelle appuyée sur une perspective socio-historique. Le travail de lecture et de production textuelle a été développé pour favoriser l'acculturation des élèves ; c'est le genre textuel du « conte étiologique » qui a été choisi comme référence commune de production pour les élèves. Le choix de ce genre s'explique non seulement parce que c'est un genre présent dans les manuels scolaires des deux pays, comme le montrent les légendes et les mythes communs au matériel didactique utilisé, mais aussi parce que ce sont de brefs récits de fiction, dont la thématique suggère une explication fantaisiste apportée aux phénomènes naturels ou aux caractéristiques des animaux ; en outre, les contes étiologiques lus impliquaient généralement des personnages et un dialogue entre eux.

Pour assurer un dispositif didactique commun aux deux classes, les enseignants ont élaboré un projet de lecture de 20 contes étiologiques éducatifs ${ }^{11}$, la plupart trouvés dans la littérature des deux pays. Les enseignants ont lu et commenté, chaque semaine pendant trois mois, une ou deux histoires aux élèves et leur ont fait produire des « contes des origines » sur thème libre ou imposé. Les élèves ont travaillé en dyades selon un protocole didactique identique dans les deux pays: pendant 10 à 15 minutes, dialogue libre pour imaginer/planifier le texte (sans prise de notes) ; puis écriture collaborative d'une demiheure pendant laquelle les élèves dictent et écrivent alternativement le texte issu du dialogue précédent, ce qui engendre de nombreuses altérations du projet initial. Durant tout le processus, les élèves sont filmés et enregistrés. Ce protocole est expérimenté depuis plus de 20 ans par l'équipe brésilienne d'Eduardo Calil $^{12}$. Il trouve sa justification dans les principes du socioconstructivisme, actuellement représenté par les travaux de J.-P. Bronckart : les interactions langagières réalisées en dyades permettent aux interactants de s'approprier des traces de l'activité langagière avant de l'intérioriser, le moteur du développement résidant dans le conflit né des confrontations inhérentes aux discussions portant sur le texte à produire. La négociation menée entre les scripteurs pour produire le texte inventé permet au chercheur de suivre l'émergence et les modifications des formes de discours rapporté final. Pour des raisons évidentes de place, l'analyse de ces interactions n'est pas présentée ici. Nous donnons seulement leur résultat à l'écrit dans les tableaux 2 et 7.

\footnotetext{
${ }^{9}$ Escola Criar e Recrear, Maceió, Alagoas, Brazil.

10 École Paul Cézanne, Marines, Val d'Oise, France.

11 Voir en Annexe I et II la liste des textes étudiés en France et au Brésil.

${ }^{12}$ La méthodologie est exposée en détail dans Autoria : a crianca e a escrita de historias inventadas, Calil (2009, 2 ème édition), Londrina, Eduel, Brésil, pp. 3-8.
} 


\section{SHS Web of Conferences}

Tableau 1. Sujets de production de textes dans les écoles brésilienne et française.

\begin{tabular}{|l|c|c|l|c|}
\hline $\begin{array}{c}\text { Séance } \\
\text { d'écriture }\end{array}$ & Pays & Date & \multicolumn{1}{|c|}{ Consigne } & $\begin{array}{c}\text { Productions } \\
\text { de textes } \\
\text { par dyades }\end{array}$ \\
\hline \multirow{2}{*}{ S1 } & FR & $10-03-11$ & $\begin{array}{l}\text { Inventer un conte des origines } \\
\text { (thème libre) }\end{array}$ & 14 \\
\cline { 2 - 6 } & BR & $3-05-11$ & $\begin{array}{l}\text { Inventer un conte des origines } \\
\text { (thème libre) }\end{array}$ & 7 \\
\hline \multirow{2}{*}{ S2 } & FR & $20-06-11$ & $\begin{array}{l}\text { Inventer un conte des origines } \\
\text { (thème libre) }\end{array}$ & 6 \\
\cline { 2 - 6 } & BR & $07-06-11$ & $\begin{array}{l}\text { Inventer un conte des origines } \\
\text { (thème libre) }\end{array}$ & 11 \\
\hline \multirow{2}{*}{ S3 } & FR & $27-06-11$ & $\begin{array}{l}\text { Inventer un conte des origines } \\
\text { ayant pour titre : } \\
\text { «Pourquoi le chien fait « ouah ouah » et } \\
\text { le chat « miaou miaou ? » }\end{array}$ & 7 \\
\cline { 2 - 6 } & BR & $19-07-11$ & $\begin{array}{l}\text { Inventer un conte des origines } \\
\text { ayant pour titre : } \\
\text { «Pourquoi le chien fait « ouah } \\
\text { ouah » et le chat « miaou miaou »? ? }\end{array}$ & 57 \\
\hline
\end{tabular}

\subsection{Hypothèse didactique}

Nous présentons ici deux types de situation d'écriture ; sur les trois séances d'écriture sélectionnées pour l'analyse, deux proposent des consignes de production laissant aux élèves la liberté d'imaginer le thème du conte étiologique. Mais dans la troisième séance d'écriture, les enseignants ont suggéré un titre pour engager les élèves à écrire des contes comportant du discours rapporté. Le titre «Pourquoi le chien fait « ouah ouah » et le chat « miaou miaou »?» suppose qu'il soit question de langage. Nous faisons donc l'hypothèse que les élèves seront ainsi amenés à produire un plus grand nombre de formes discursives attribuées aux personnages animaux.

Dans la sélection ainsi produite, 57 textes d'élèves ont été recueillis, 20 produits par les élèves de l'école brésilienne et 37 par ceux de l'école française. Tous les textes ayant été écrits par dyades, chaque occurrence de production de textes mentionnée dans le tableau (dernière colonne de droite) doit donc être comprise comme le résultat du travail de deux scripteurs. Exemple : 14 textes français pour 27 élèves (classe en nombre impair, un élève travaille seul) ; 7 textes brésiliens pour 14 élèves. Les fluctuations de nombre observées dans la dernière colonne du tableau ci-dessous résultent d'absences occasionnelles d'élèves dans la classe : nous travaillons en situation naturelle, non de façon expérimentale.

\subsection{Terminologie conceptuelle}

Nous avons dû faire des choix théoriques afin d'appréhender les structures grammaticales servant de support au DR. Le cadre auquel nous nous référons est celui de J. Authier-Revuz (voir point précédent). Cependant le travail théorique de la linguiste vise à une description du champ du « discours autre » en langue ; c'est pourquoi elle considère comme sans conséquence pour la théorie les manifestations non-standard du DR essayées par les apprenants. Pour nous, au contraire, les différentes structures empiriques que nous avons classées sont l'occasion de montrer la précocité et la richesse des réalisations de DR chez de jeunes scripteurs suivant le degré de complexité mis en œuvre pour les incorporer au récit. C'est donc le nombre et la variété des formes de DR employées en discours que nous avons cherché à inventorier. 
Tableau 2. Enoncés de discours rapporté (EDR) et types de structures.

\begin{tabular}{|l|l|}
\hline \multicolumn{2}{|c|}{ Types de structures d' EDR } \\
\hline 1. & Verbe introducteur dicendi ${ }^{15}+$ discours indirect (VID+DI) \\
\hline 2 & Verbe introducteur dicendi + discours narrativisé(VID+DN) \\
\hline 3. & Verbe introducteur dicendi + discours direct (VID+DD) \\
\hline 4. & Verbe introducteur dicendi + que + discours indirect (VID+que+DI) \\
\hline 5. & Verbe introducteur dicendi + discours indirect libre(VID+DIL) \\
\hline 6. & Verbe introducteur sentiendi + discours indirect(VIS+DI) \\
\hline 7. & Verbe introducteur sentiendi + discours narrativisé (VIS+DN) \\
\hline 8. & Verbe introducteur sentiendi + discours direct(VIS+DD) \\
\hline 9. & Sans verbe introducteur + discours direct(s/VI+DD) \\
\hline 10. & Discours direct (DD) \\
\hline 11. & Discours direct + incise avec verbe dicendi $($ DD+iVD) \\
\hline 12. & Discours direct + incise avec verbe dicendi+ discours direct (DD+iVD+DD) \\
\hline 13. & Discours du narrateur (DDn) \\
\hline 14. & Discours indirect libre (DIL) \\
\hline
\end{tabular}

Nous considérons comme actes d'énonciation les manifestations qui sont « du discours sur du discours et du discours dans du discours » pour reprendre les propos de [34] Volochinov (1977) ${ }^{13}$, ce qui nous a amenés à intégrer provisoirement les manifestations du narrateur hors de son récit. Nous nommons « énoncés de discours rapporté » (EDR) les structures relevées chez ces jeunes scripteurs qui sont à la fois attestées énonciativement et transitoires. Nous avons ainsi identifié 14 types d'EDR dans les productions tant françaises que brésiliennes ${ }^{14}$.

Nous avons quantifié ces 14 types d'EDR, en comparant leurs occurrences dans les productions de textes des deux pays, afin de pouvoir observer leurs similitudes et leurs différences.

\section{Observations et analyses}

\subsection{Résultats quantitatifs en nombre de mots}

Une première observation se réfère à la quantité de mots par texte ${ }^{16}$.

Les premiers résultats quantitatifs font apparaitre des différences dans la longueur des productions. Le nombre total de productions françaises (désormais FR) est presque le double des productions brésiliennes (désormais BR) en raison de la différence du nombre d'élèves dans chaque classe. Toutefois, les élèves brésiliens écrivent des textes plus longs que les élèves français : dans tous les cas de figure, la moyenne et la médiane sont plus élevées dans les textes brésiliens ; la différence entre le nombre total moyen de mots écrits est de $26,6 \%$, soit une moyenne de 100,2 mots pour les trois séries de textes BR, et de 73,6 mots pour les textes FR.

Isolé, ce résultat n'a cependant aucune valeur significative, à moins d'être rapproché de ce qui nous intéresse dans cette recherche : la présence de discours représentant les mots d'autrui, ce que nous avons identifié comme une manifestation de la capacité du sujet à dialoguer avec le mot d'autrui, capacité entrant dans l'habileté à écrire des histoires.

\footnotetext{
13 L'auteur signifie ainsi qu'il s'agit d'énoncés à la fois réflexifs et enchâssés ou incorporés.

14 Voir en Annexe III le tableau présentant ces structures avec des exemples.

15 La distinction entre verbes dicendi et verbes sentiendi ou plus largement les verbes exprimant des procès mentaux a été discutée par [35] Rabatel (2003). La distinction est ici schématique. Elle oppose les verbes de parole à ceux pour lesquels sont inférés des actes de parole, ce qui suppose une part de lecture interprétative.

16 On rappelle que chaque texte est issu de l'écriture d'une dyade et non d'un seul scripteur.
} 


\section{SHS Web of Conferences}

Tableau 3. Nombre de mots par consigne dans les textes BR et FR (tous les textes).

\begin{tabular}{|c|c|c|c|c|c|c|c|c|}
\hline \multicolumn{7}{|c|}{ Mots par consigne (C) dans les textes BR et FR (tous les textes) } \\
\hline & \multicolumn{3}{|c|}{ BR } & \multicolumn{3}{c|}{ FR } \\
\cline { 2 - 9 } & Textes & Mots & Moyenne & Médiane & Textes & Mots & Moyenne & Médiane \\
\hline C1 & 7 & 565 & 80,7 & 77 & 14 & 871 & 62,2 & 72 \\
\hline C2 & 6 & 599 & 99,8 & 85 & 12 & 953 & 79,4 & 74,5 \\
\hline C3 & 7 & 841 & 120,1 & 99 & 11 & 872 & 79,2 & 75 \\
\hline TT & 20 & 2005 & 100,2 & 87 & 37 & 2696 & 73,6 & 74,5 \\
\hline
\end{tabular}

Tableau 4. Nombre de mots par consigne dans les textes BR et FR (textes avec DR).

\begin{tabular}{|c|c|c|c|c|c|c|c|c|}
\hline \multicolumn{7}{|c|}{ Mots par consigne (C) dans les textes BR et FR (seulement les textes avec DR) } \\
\hline & \multicolumn{3}{|c|}{ BR } & \multicolumn{4}{c|}{ FR } \\
\cline { 2 - 9 } & Textes & Mots & Moyenne & Médiane & Textes & Mots & Moyenne & Médiane \\
\hline C1 & 3 & 293 & 97,6 & 82 & 4 & 331 & 82,7 & 79,5 \\
\hline C2 & 6 & 599 & 99,8 & 65 & 6 & 516 & 86 & 84,5 \\
\hline C3 & 7 & 931 & 133 & 100 & 10 & 606 & 60,6 & 59 \\
\hline TT & $\mathbf{1 6}$ & $\mathbf{1 8 2 3}$ & $\mathbf{1 1 0 , 1}$ & $\mathbf{8 2 , 3}$ & $\mathbf{2 0}$ & $\mathbf{1 4 5 3}$ & $\mathbf{7 6 , 4}$ & $\mathbf{7 9 , 5}$ \\
\hline
\end{tabular}

La présence du DR est attestée dans plus de la moitié des textes, à la fois dans les productions françaises et brésiliennes. Mais les chiffres des productions brésiliennes sont significatifs : sur les 34 productions réalisées, seules 8 n'ont aucune forme de DR. En termes de pourcentage, cela signifie que le DR est présent dans 76,4 \% des histoires inventées par les élèves brésiliens. Dans le groupe des productions françaises, il y a aussi une plus grande quantité de textes comportant du DR, mais la différence de pourcentage entre les textes avec DR et ceux sans DR est nettement plus faible : $54 \%$ des manuscrits avec DR et $45,9 \%$ sans DR.

Cependant, quand on n'envisage que les productions dans lesquelles figure du discours rapporté, on constate que, du côté brésilien comme du côté français, le nombre de mots augmente globalement : il passe de 100,2 à 110,1 pour les BR, de 73,6 à 76,4 pour les FR, ce que montre le tableau 4.

\subsection{Résultats quantitatifs et tâches de production}

Nous avons voulu également mettre en rapport les résultats quantitatifs avec les tâches de production et les consignes qui les accompagnent.

Tout d'abord, il est intéressant d'observer que le DR apparaît dans toutes les tâches de production analysées, mais avec des valeurs qui varient beaucoup quantitativement.

Considérons les productions françaises. Si l'on fait la somme du nombre d'énoncés de DR apparaissant dans les deux premières tâches de production $(\mathrm{C} 1 \& \mathrm{C} 2)$ on arrive aux résultats de 17 énoncés de DR pour la $1^{\text {ère }}$ consigne et 16 pour la 2ème consigne (conte des origines à thème libre toutes les deux). Mais si l'on compare les données avec celles de la $3^{\text {ème }}$ tâche de production (consigne à thème imposé) celle-ci représente à elle seule, en nombre d'occurrences, plus du double des deux autres.

Quant aux énoncés de DR brésiliens, bien qu'ils soient en nombre plus important, ils suivent une courbe comparable à celle des énoncés français, le nombre d'énoncés d'EDR réalisés lors de la $3^{\text {ème }}$ tâche de production représentant la somme des deux autres.

Le schéma suivant permet de visualiser l'évolution des deux courbes.

On constate ainsi que se vérifie l'hypothèse didactique: une consigne inductrice entraîne un nombre accru de paroles de personnages. Reste à examiner dans quelles structures linguistiques elles sont insérées. 


\section{Quantité DR par consigne BR/FR}

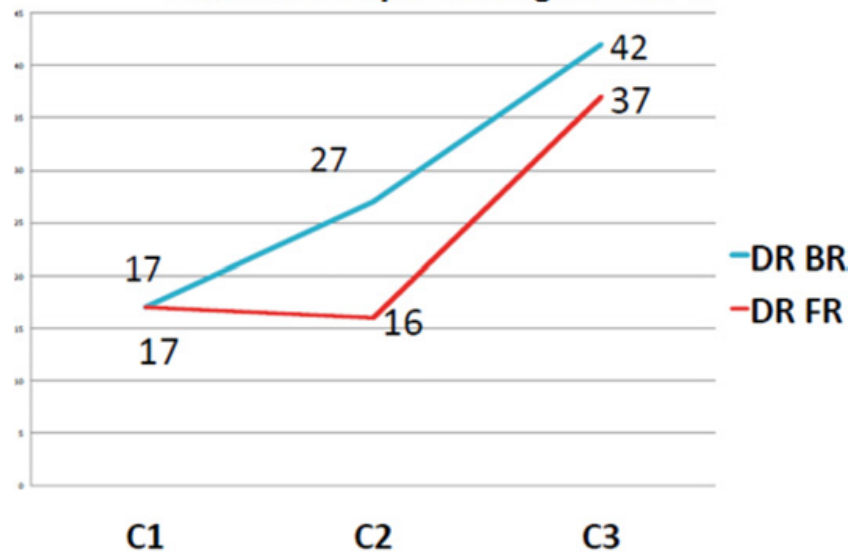

Schéma 1. Quantité d'occurrences de DR (EDR) par consigne dans les textes brésiliens et français.

\section{Structures de DR et types de verbes introducteurs dans les textes BR et FR}

Les formes d'apparition de DR sont très diverses, même chez des scripteurs débutants. Les 14 types que nous avons identifiés (avec des variantes comme la présence de gérondif ou modalisations verbales dans les productions écrites françaises) se répartissent ainsi dans les productions françaises et brésiliennes :

\subsection{Analyse de la répartition des types de structures dans les textes français et brésiliens}

On remarque que les seules structures présentes dans les textes français qui n'ont pas été utilisées en portugais sont des exemples de discours indirect libre (type 14) ; en revanche, dans les textes français, pas d'incise (types 11 et 12) ni de discours indirect avec « que » (type 4) pourtant fort connus des élèves en lecture. On voit donc ici se dessiner des différences d'emploi.

Il y a cependant des similitudes dans le choix prédominant d'un type de structure.

Si l'on considère les types de structure ayant la fréquence la plus élevée, on relève en effet une coïncidence. Dans les deux séries il y a une incidence élevée du type 3 (VID + DD) : avec 39 occurrences pour les textes BR et 37 pour les manuscrits FR, et du type 10 (DD), avec 16 occurrences pour BR et 8 pour FR.

Ces indicateurs donnent à penser que, tant pour les élèves brésiliens que pour les élèves français, le type le plus commun du discours rapporté est le DD, précédé ou non d'un verbe introducteur dicendi. Ce résultat confirme les conclusions de nombreux chercheurs depuis l'étude de [13] Hickmann (1991) [15] Ferreiro et al. (1996) et en didactique pour l'écrit : [19-21] Boré (2004a, 2004b, 2009a). Mais il concerne ici des données écrites et en contraste de langue, obtenues dans des conditions de production naturelles. 

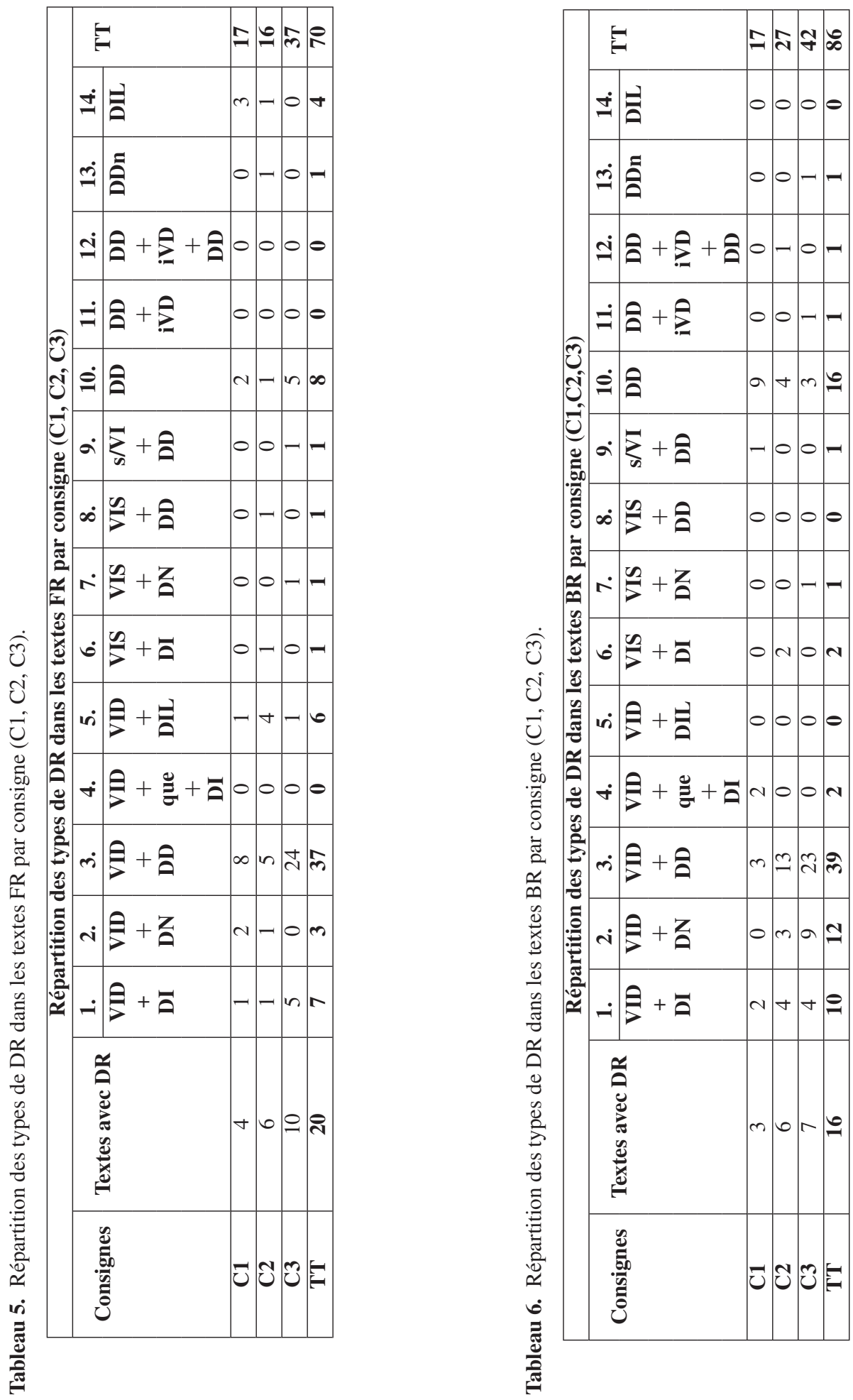
Tableau 7. Types d'occurrences lexicales de VIDR dans les textes BR et FR (verbes dicendi \& sentiendi).

\begin{tabular}{|c|c|c|c|}
\hline $\mathbf{N}^{\circ}$ & 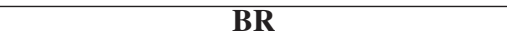 & $\mathbf{N}^{\circ}$ & FR \\
\hline 1. & $\begin{array}{l}\text { ficar com muita raiva (com o sentido de } \\
\text { « esbravejar ») }\end{array}$ & & \\
\hline \multirow[t]{2}{*}{2.} & dar um grito de guerra & 1. & crier \\
\hline & 3. desculpar-se & & \\
\hline 4. & fazer (com o sentido de « falar ») & 2. & faire \\
\hline 5. & anunciar & & \\
\hline 6. & brigar & 3. & se disputer \\
\hline 7. & chorar & 4. & pleurer de \\
\hline 8. & falar & & \\
\hline 9. & decidir & 5. & décider \\
\hline 10. & dizer & 6. & dire \\
\hline 11. & comunicar-se & & \\
\hline 12. & $\begin{array}{l}\text { dar uma dica }(\text { com o } \text { ontido de } \\
\text { « sugerir ») }\end{array}$ & & \\
\hline \multirow[t]{2}{*}{13.} & expulsar & & \\
\hline & & 7. & vouloir (avoir l'intention de) \\
\hline 14. & chamar & 8. & appeler \\
\hline \multirow[t]{6}{*}{15.} & implorar & & \\
\hline & & 9. & $\begin{array}{l}\text { en avoir assez de } \\
\text { (verbe sentiendi supposant des paroles) }\end{array}$ \\
\hline & & 10. & en avoir marre (même chose) \\
\hline & & 11. & imaginer (verbe de cognition) \\
\hline & & 12. & croire (verbe de cognition) \\
\hline & & 13. & s'apercevoir (verbe de cognition) \\
\hline 16. & mandar & & \\
\hline 17. & ordenar & & \\
\hline 18. & pedir & 14. & demander (une permission) \\
\hline 19. & pensar & 15. & se dire (au sens de « penser ») \\
\hline 20. & perguntar & 16. & demander \\
\hline 21. & reclamar & 17. & ne pas être d'accord \\
\hline 22. & responder & 18. & répondre \\
\hline \multirow[t]{2}{*}{23.} & fazer un accordo em libras & & \\
\hline & & 19. & écrire une lettre (disant) \\
\hline 24. & rezar (prier) & & \\
\hline 25. & $\begin{array}{l}\text { ter uma ideia (com o sentido de } \\
\text { «pensar ») }\end{array}$ & 20. & trouver une idée \\
\hline
\end{tabular}

\subsection{Variété lexicale des verbes introducteurs de discours rapporté (VIDR)}

Nous avons aussi cherché à savoir si la variété des structures s'accompagnait d'une variété lexicale des verbes introducteurs. Nous avons identifié 25 types d'occurrences dans les textes brésiliens et 20 dans les manuscrits français, avec des créations intéressantes dans les deux langues.

Il y a certes une différence numérique dans la variété lexicale des verbes introducteurs de DR brésiliens et français. Mais le plus intéressant est le nombre d'équivalences : 13 verbes sont lexicalement communs ou équivalents dans les deux langues; ils sont surlignés en jaune dans le tableau 7. La comparaison vaut surtout qualitativement car les constructions syntaxiques diffèrent dans les deux langues pour des occurrences apparemment comparables : ainsi «parler » n'admet pas de DD ni de «DI + que » en français, contrairement au portugais « falar » de construction beaucoup plus souple. On 
ne peut donc pas faire d'inférences sur la plus ou moins grande richesse lexicale d'emploi des verbes par les élèves à partir d'un simple décompte quantitatif.

\section{Conclusion}

Des indications significatives semblent se dégager de cette étude comparative.

1. Sur le plan quantitatif, nous constatons d'abord une tendance chez les élèves brésiliens à écrire des textes d'invention plus longs que les élèves français avec une moyenne de $26,6 \%$ de mots en plus. Ce résultat coïncide par ailleurs avec un nombre d'occurrences de discours rapportés deux fois plus élevé chez les élèves brésiliens que chez les élèves français (80\% des textes brésiliens en comportent contre $54,1 \%$ des textes FR). Si nous comparons alors le nombre de mots présents seulement dans les productions qui comportent des discours rapportés, en France et au Brésil, nous constatons que l'écart se resserre quant au nombre de mots. Ces résultats semblent ainsi indiquer que le travail d'invention est alimenté et augmenté par la présence de discours rapportés. Toutefois l'augmentation globale en nombre de mots ne se vérifie pas au niveau des moyennes dans les textes français pour la dernière consigne d'écriture : s'il y a incontestablement plus d'élèves qui ont utilisé du DR au cours de la dernière consigne de production (C3), ils n'ont pas pour autant utilisé plus de mots : ce qui a pu entrainer un nombre de mots plus élevé globalement que lors de la 2ème consigne (car plus d'élèves ont utilisé du DR), mais une moyenne plus faible car ces nouveaux utilisateurs de DR écrivent des textes plus courts.

2. Sur le plan qualitatif, l'étude montre des différences à la fois dans la variété et la nature sémantique des verbes introducteurs des deux pays. Cependant nous constatons un emploi dominant du verbe dire chez les élèves brésiliens et français, et surtout un type de structure de discours rapporté dominant dans les deux pays : celle du discours direct avec verbum dicendi. Celle-ci se retrouve encore plus massivement dans le dernier exercice recueilli (C3) dont le titre de la consigne ${ }^{17}$ induisait un discours direct : cela pourrait expliquer la présence des 23 et 24 occurences de DR avec la structure VID + DD respectivement. dans les textes brésiliens et français au cours de cette dernière séance de production à titre imposé. L'hypothèse semble s'accorder avec le cadre théorique que nous avons élaboré : la structure dialogale du DD représenté est une réminiscence du discours intérieur dialogique qui permet à l'enfant de créer un univers fictionnel, au moins pour les enfants de cet âge. Ces points doivent cependant être confirmés par des études futures.

3. Pour les contes étiologiques réunis dans les corpus, nous avons vu que ce sont surtout les structures syntaxiques lexicales et sémantiques des verbes introducteurs qui devraient être systématiquement étudiées et croisées avec les types de verbes introducteurs utilisés par les élèves, afin de connaître précisément les ressources dont disposent les élèves des deux pays sur le plan linguistique. Néanmoins, en faisant l'analyse des verbes introducteurs et des structures de discours rapporté dans le corpus de référence des textes français (Annexe I), nous avons constaté que, comme dans tous les contes, la structure verbe dire + DD est certes la plus fréquente, mais que beaucoup d'autres structures sont aussi représentées. Il ne semble donc pas productif de postuler un lien de causalité direct entre visée explicative du conte étiologique et emploi d'un type de DR. En revanche tous les textes du corpus de contes auquel ont eu accès les élèves comportaient du DR, alors que les scripteurs n'ont pas tous utilisé de DR dans leurs productions. Il ne semble donc pas que l'emploi du DR soit dû prioritairement au modèle générique proposé en lecture aux élèves mais plutôt, comme l'ont montré les résultats dans le schéma 1, au cadre didactique suggérant son emploi, à savoir un titre inducteur et la possibilité d'envisager le point de vue de l'autre par l'exercice du dialogue en dyades.

17 « Pourquoi le chien dit « ouah, ouah!» et le chat « miaou, miaou !». 
Les résultats précédents ne font qu'ouvrir à la discussion un ensemble de champs et d'hypothèses peu explorés dont le présent article donne un premier aperçu.

\section{Références}

[1] C. Bonnet \& J. Tamine-Gardes. Quand l'enfant parle du langage, Bruxelles, Mardaga. (1984)

[2] L. Lentin, et alii. Recherches sur l'acquisition du langage, Presses de la Sorbonne Nouvelle, I et II. (1984 et 1988)

[3] L. Lentin. Apprendre à penser, parler, lire, écrire. Acquisition du langage oral et écrit. Paris, E.S.F. (2009)

[4] J. Bernicot, E. Veneziano et al. Interactions verbales et acquisitions du langage, Paris, L'Harmattan. (2010)

[5] E. Goodell, \& J. Sachs. Direct and Indirect Speech in English-Speaking Children'sRetold Narratives, Discourse Processes 15 (4), 395-422, (1992)

[6] J. Simon. La langue écrite de l'enfant. Paris : Presses Universitaires de France (1973)

[7] B. Schneuwly. Le langage écrit chez l'enfant. Paris : Delachaux-Niestlé (1988)

[8] M. Fayol. Le récit et sa construction, une approche de psychologie cognitive, Paris, Delachaux \& Niestlé (1985)

[9] M. Fayol. Des idées au texte, psychologie cognitive de la production verbale, orale et écrite, PUF (1997)

[10] M.M. de Gaulmyn, Les verbes de communication dans la structuration du discours. Essai sur la réflexivité du langage. Récits d'enfants et échanges entre enfants et adultes. Thèse de doctorat d'État, Paris VIII (1983)

[11] J.-N. Foulin, et M. Fayol. Etude en temps réel de la production écrite chez des enfants de sept et huit ans. European Journal of Psychology of Education, 3, 461-475 (1988)

[12] M. Hickmann, Le discours rapporté : aspects méta-pragmatiques du langage et de son développement. Bulletin de Psychologie, Numéro spécial : «Les processus de contrôle dans la résolution de tâches complexes ». Tome XLIV, No. 399, 121-137, (1991)

[13] N. Dittmar, \& A. Reich (éds) Modality in Language Acquisition / Modalité et Acquisition Des Langues, De Gruyter. (1993)

[14] E. Ferreiro, Cl.Pontecorvo, Ribeiro Moreira et Garcia Hidalgo (éds) Chapeuzinho Vermelho aprende a escrever: estudos psicolinguísticos comparativos em três línguas. San Paulo: Atica, (1996)

[15] J. B. Bosco Figueiredo, A reprodução de enunciação indireta em narrativas infantis. Rev. de Letras 20 - Vol. 1/2 - jan/dez. 91-102, (1998)

[16] M. Elias Soares. "O desenvolvimento da habilidade de organização textual em língua escrita" Letras de Hoje, Porto Alegre, v. 34, n.3, 101-110 (1999)

[17] C. Granget. Le développement du discours rapporté en français L2, Acquisition et Interaction en Langue Etrangère 26, 89-112 (2008)

[18] Cl. Fabre. Variantes de réécriture, citations, discours rapportés à l'école élémentaire. Cahiers d'Acquisition et de Pathologie du Langage, Fascicule 9, «Réécriture et interactivité », Université René-Descartes (1992)

[19] C. Boré. L'écriture scolaire : langue, norme, "style" : quelques exemples dans le discours rapporté, Linx 51, 91-106 (2004a)

[20] C. Boré. Discours rapportés dans les brouillons d'élèves : vrai dialogisme pour une polyphonie à construire, Pratiques 123-4, Université de Metz, 143-169 (2004b)

[21] C. Boré. Le dialogue de fiction scolaire, moteur de l'invention dans Dufays et Plane (éds) L'écriture de fiction en classe de français, Presses Universitaires de Namur, 189-210 (2009)

[22] C. Boré. Modalités de la fiction dans l'écriture scolaire. Paris, I'Harmattan (2010) 


\section{SHS Web of Conferences}

[23] S. Plane, F. Rondelli \& C. Vénérin. Variations, fidélité, infidélité : l'écriture et la réécriture de discours rapportés par de jeunes scripteurs. In C. Dessoutter \& C. Mellet (Dir.) Il Discorso Reportato e le sue marche: Prospettive teoriche e didattiche. (sous presse 2013)

[24] L.S. Vygotski. Pensée et langage. Paris : La Dispute (traduction F. Sève) [1934, 1997]

[25] J.P. Bronckart. Activité langagière, textes et discours. Pour un interactionisme socio-discursif, Paris, Delachaux-Niestlé (1997)

[26] Bruner, J. (trad. Y. Bonin). L'éducation, entrée dans la culture : Les problèmes de l'école à la lumière de la psychologie culturelle, Paris, Retz (2008)

[27] P. Harris, L'imagination chez l'enfant. Paris : Retz [2000] (2007)

[28] A. Reboul. Communication, fiction et expression de la subjectivité, Langue française 128, 9-29 (2000)

[29] M. Bakhtine. Du discours romanesque, dans Esthétique et théorie du roman. Paris, Gallimard, 83-233 [1934, 1975] (1978)

[30] J. Authier-Revuz. Ces mots qui ne vont pas de soi, boucles réflexives et non-cö̈ncidences du dire, Paris : Larousse, Limoges, Lambert-Lucas (1995, rééd 2013)

[31] J. Bres, \& A. Nowakowska. Dialogisme : du principe à la matérialité discursive, dans L. Perrin (Dir.), Le sens et ses voix, Recherches linguistiques 28, Metz: Université de Metz, 21-48 (2006)

[32] E. Calil, \& A. De Re. Analyse d'une histoire inventée multimodale : le cas de l'onomatopée visuelle. Anpoll Magazine, vol. 2, 12-38 (2009)

[33] E. Calil \& C. Boré. Les formes de discours rapporté dans les récits de fiction écrits par des élèves brésiliens ef français, Congreso Internacional de la Asociacion de Linguistica y la philologie Amérique latine (Alfal), 2011, Alcala de Henares. XVI Congreso Internacional de la Alfal. Alcala de Henares: Universidad de Alcala, 2807-2815 (2011)

[34] V. N. Voloshinov. Le marxisme et la philosophie du langage, traduction et Patrick Seriot Inna Tylkowski-Ageeva, édition bilingue, Chapitres III et IV, Limoges : Lambert-Lucas, 385-466. $[1929,1930](2010)$

[35] A. Rabatel. Les verbes de perception en contexte d'effacement énonciatif : du point de vue représenté aux discours représentés, Travaux de linguistique 1 no 46, 49-88 (2003) 


\section{ANNEXE I}

Index des contes étiologiques ${ }^{18}$ lus en France.

\begin{tabular}{|c|c|}
\hline $\begin{array}{l}\text { 1. Comment sont apparusles nuages } \\
\text { Esquimsuxd'Asie }\end{array}$ & $\begin{array}{l}\text { 11. Pourquoiles crabesn'ont pas de tête } \\
\text { Guadeloupe }\end{array}$ \\
\hline $\begin{array}{l}\text { 2. Pourquoiles poissons vivent dans } \\
\text { leau } \\
\text { Sibérie }\end{array}$ & $\begin{array}{l}\text { 12. Le chameauet sa bosse } \\
\text { R. Kipling, trad. Griead }\end{array}$ \\
\hline $\begin{array}{l}\text { 3. L'enfant auserpent } \\
\text { Cotte d'Ivoire }\end{array}$ & $\begin{array}{l}\text { 13. Pourquoill ne faut pas battre les } \\
\text { enfants } \\
\text { Conte Inca }\end{array}$ \\
\hline $\begin{array}{l}\text { 4. La légende de l'escargot } \\
\text { Burkina-Faso }\end{array}$ & $\begin{array}{l}\text { 14. L'étoile et le nénuphar } \\
\text { Amerique du Nord (Indien, reecrit par Philippe Soupault) }\end{array}$ \\
\hline $\begin{array}{l}\text { 5. Les premièreslarmes et les taches } \\
\text { surla lune } \\
\text { Algerie }\end{array}$ & $\begin{array}{l}\text { 15. Le corbeau et le soleil } \\
\text { Conte du Grand-Nord }\end{array}$ \\
\hline $\begin{array}{l}\text { 6. Quelle forme avaientles premiers } \\
\text { hommes } \\
\text { Espagne }\end{array}$ & $\begin{array}{l}\text { 16. Pourquoile putois sent mauvais } \\
\text { Afrique du Sud }\end{array}$ \\
\hline $\begin{array}{l}\text { 7. Les baies d'amour } \\
\text { Amerique du Nord (Cherokee) }\end{array}$ & $\begin{array}{l}\text { 17. La lune et sa mére } \\
\text { D'sprès Grimm }\end{array}$ \\
\hline $\begin{array}{l}\text { 8. Le cocotier } \\
\text { Philippines }\end{array}$ & $\begin{array}{l}\text { 18. Commentse fait-il que quily ait des } \\
\text { chauves-souris? } \\
\text { Espsgne. }\end{array}$ \\
\hline $\begin{array}{l}\text { 9. Pourquoile ciel est loin maintenant } \\
\text { Nigeria }\end{array}$ & $\begin{array}{l}\text { 19. Pourquoilly a des hommes chauves } \\
\text { cóte ólvoire }\end{array}$ \\
\hline $\begin{array}{l}\text { 10. Pourquoiles tortues vivent dans } \\
\text { l'eau } \\
\text { Nouvelle-Calédonie }\end{array}$ & $\begin{array}{l}\text { 20. Comment sont nés les premiers } \\
\text { hommes? } \\
\text { Brésil (Amszonie) }\end{array}$ \\
\hline
\end{tabular}

18 La plupart de ces textes proviennent du livre de Muriel Bloch, 365 contes des pourquoi et des comment, Gallimard-Jeunesse -Giboulées, 1997. 
ANNEXE II

Index des contes étiologiques lus au Brésil.

\begin{tabular}{|c|c|}
\hline $\begin{array}{l}\text { 1. La Fête dansle ciel } \\
\text { Braguinha }\end{array}$ & $\begin{array}{l}\text { 11. Pourquoile zébre est rayé } \\
\text { Barbosa. }\end{array}$ \\
\hline $\begin{array}{l}\text { 2. Comment est né le monde et } \\
\text { (presque) tout ce qui est dessus } \\
\text { Zatz. }\end{array}$ & $\begin{array}{l}\text { 12. Pourquoile caméléon change de } \\
\text { couleur } \\
\text { Barbosa. }\end{array}$ \\
\hline $\begin{array}{l}\text { 3. Le bec du Toucan } \\
\text { Zatz. }\end{array}$ & $\begin{array}{l}\text { 13. Pourquoile porc vit dans une } \\
\text { porcherie } \\
\text { Barbosa. }\end{array}$ \\
\hline $\begin{array}{l}\text { 4. Pourquoila moufette pue } \\
\text { Zatz. }\end{array}$ & $\begin{array}{l}\text { 14.Amis, mais pas pour toujours } \\
\text { Barbosa }\end{array}$ \\
\hline $\begin{array}{l}\text { 5. Commentsont apparuesles } \\
\text { chauves-souris } \\
\text { Zatz. }\end{array}$ & $\begin{array}{l}\text { 15. Pourquoi les pintades ont des points } \\
\text { blancs } \\
\text { Barbosa }\end{array}$ \\
\hline $\begin{array}{l}\text { 6. Pourquoi en péchantici oulà, } \\
\text { les poissons vivaient dans la } \\
\text { mer } \\
\text { Zatz. }\end{array}$ & $\begin{array}{l}\text { 16. L'autruche (pourquoile mâle couve } \\
\text { les œufs) } \\
\text { Lisboa }\end{array}$ \\
\hline $\begin{array}{l}\text { 7. Pourquoiles crabes n'ont pas de } \\
\text { tête } \\
\text { Zatz. }\end{array}$ & $\begin{array}{l}\text { 17. Pourquoila girafe n'a pas de voix } \\
\text { Barbosa. }\end{array}$ \\
\hline $\begin{array}{l}\text { 8. Commentle lièvre a atterri sur la } \\
\text { lune } \\
\text { Zatz. }\end{array}$ & $\begin{array}{l}\text { 18. Pourquoiles singes se cachent } \\
\text { dansles arbres } \\
\text { Barbosa }\end{array}$ \\
\hline $\begin{array}{l}\text { 9. Comment sont apparuesles } \\
\text { langues } \\
\text { Zatz }\end{array}$ & $\begin{array}{l}\text { 19. Commentles premiers hommes sont } \\
\text { nés } \\
\text { Lodv }\end{array}$ \\
\hline $\begin{array}{l}\text { 10. Pourquoile lapin a de longues } \\
\text { oreilles } \\
\text { Zatz }\end{array}$ & $\begin{array}{l}\text { 20. Pourquoile chien est l'ennemidu } \\
\text { chat... et le chat du rat } \\
\text { cascudo. }\end{array}$ \\
\hline
\end{tabular}




\section{ANNEXE III}

Discours rapporté et types de structures avec exemples.

\begin{tabular}{|c|c|c|c|}
\hline \multicolumn{4}{|c|}{ Discours rapporté et types de structures } \\
\hline $\mathrm{Ma}$ & $\mathrm{BR}$ & $\mathrm{Ma}$ & FR \\
\hline 1. & \multicolumn{3}{|c|}{ Verbo introducteur dicendi + discours indirect(VID+DI) } \\
\hline $001 \mathrm{BR}$ & $\begin{array}{c}\text { a baleia pediu comida ao tubarão. } \\
\text { La baleine a demandé de la nourriture } \\
\text { au requin }\end{array}$ & $016 \mathrm{FR}$ & $\begin{array}{l}\text { le petit enfant demande [à sa mère] síl } \\
\text { pouvait devenir président. }\end{array}$ \\
\hline 2. & \multicolumn{3}{|c|}{ Verbo introdutor dicendi + Discours narrativisé (VID+DN) } \\
\hline 009BR & $\begin{array}{c}\text { o gato fez um acordo em libras } \\
\text { com / a senhorita zebra. } \\
\text { Le chat a écrit un accord avec } \\
\text { mademoiselle Zèbre }\end{array}$ & $011 \mathrm{FR}$ & $\begin{array}{l}\text { et la lune commence à écrire une lettre } \\
\text { d'amoure. }\end{array}$ \\
\hline 3. & \multicolumn{3}{|c|}{ Verbo introducteur dire+ discours direct (VID+DD) } \\
\hline $017 \mathrm{BR}$ & $\begin{array}{l}\text { e o cachorro disse [ao gato]-você } \\
\text { quer me deixar com fome? } \\
\text { Et le chien dit [au chat ]: tu vevx me } \\
\text { laisser mourir de faim? }\end{array}$ & $016 \mathrm{FR}$ & le président dit [à l'enfant] : tu est trop petit! \\
\hline 4. & \multicolumn{3}{|c|}{ Verbo introdutor dicendi + que + discours indirect (VID + que+DI) } \\
\hline 002BR & $\begin{array}{l}\text { aí, ela [a sra. Tartaruga] disse que só } \\
\quad \text { /com a estratégia do sol. } \\
\text { Là, elle [Madame la Tortue] a dit } \\
\text { qu'[il fallait] Jeulements'entendre } \\
\text { avec le soleil }\end{array}$ & $\varnothing$ & $\varnothing$ \\
\hline 5. & \multicolumn{3}{|c|}{ Verbo introdutor dicendi + discours indirect libre (VID+DIL) } \\
\hline$\varnothing$ & $\varnothing$ & $021 \mathrm{FR}$ & $\begin{array}{c}\text { Il était une fois une femme qui n'avait pas } \\
\text { d'enfant et / voulait des animaux comme } \\
\text { enfants }\end{array}$ \\
\hline 6. & \multicolumn{3}{|c|}{ Verbe introducteur dicendi + discours indirect avec infinitif (VID+DI+inf) } \\
\hline 008BR & $\begin{array}{l}\text { [mas, não iriam aguentar ficar sem } \\
\text { comer] e o rei o leão/ordenou fazer } \\
\text { isso. } \\
\text { [Mais ne supportait pas d'être sans } \\
\text { nourriture] et le roi lion/a ordonné } \\
\text { de le faire. }\end{array}$ & OSFR & Et ils/ dessidaire de former une famille \\
\hline 7. & \multicolumn{3}{|c|}{ Verbe introducteur sentiendi $=$ discours narrativisé sans complément (VID+DN) } \\
\hline 009BR & $\begin{array}{l}\text { a zebra ficou com muita / raiva do } \\
\text { gato. } \\
\text { Le zèbre se mit en colère contre le } \\
\text { chat }\end{array}$ & 029FR & Et après, le chat et / le chien se disputait. \\
\hline 8. & \multicolumn{3}{|c|}{ Verbo introducteur dicendi + discours direct (VID+DD) } \\
\hline$\varnothing$ & $\varnothing$ & 023FR & $\begin{array}{c}\text { [et un animal avait tremper sa patte dans } \\
\text { l'eau] et cria - l'eau est bonne }\end{array}$ \\
\hline 9. & \multicolumn{3}{|c|}{ Sans verbe introducteur + discours direct $(\mathrm{s} / \mathrm{VI}+\mathrm{DD})$} \\
\hline 002BR & $\begin{array}{l}\text { [borboleta falando para a sra. } \\
\text { Tartaruga] aí, ela - como / vou } \\
\text { alcançar o sol se não sei voar.? / Se } \\
\text { tambêmnão tenho asas e a senhora } \\
\text { não/ sabe colocá-las.? } \\
\text { [le papillon parlant à Mme la } \\
\text { Tortue] et lui: - comment /vous } \\
\text { allez atteindre le soleil si vous ne } \\
\text { volez pas? / Et aussi si vous n'avez } \\
\text { pas d'ailes et si vous ne savez pas } \\
\text { les mettre? }\end{array}$ & 034FR & $\begin{array}{l}\text { [le narrateur imitant le chat } \\
\text { ] et - miaou, miaou. }\end{array}$ \\
\hline
\end{tabular}




\begin{tabular}{|c|c|c|c|}
\hline 10. & \multicolumn{3}{|c|}{ Verbe dire+ Discours direct (DD) } \\
\hline+ & $\begin{array}{l}\text { [leão perguntando para sua mãe] } \\
\text { - tá na hora do almoço, já, mamãe? } \\
\text { [Le lion demandant à sa mère] } \\
\text {-c'est l"heure de déjeuner } \\
\text { maintenant maman? }\end{array}$ & $014 \mathrm{FR}$ & [la mère dit à l'. enfant] Viens goûter!!!! \\
\hline 11. & & & \\
\hline 016BR & $\begin{array}{l}\text { [cachorro respondendo para o gato] } \\
\text { está certo - disse o cachorro. } \\
\text { [le chien répondant au chat ] -c'est } \\
\text { vrai, dit le chien. }\end{array}$ & $\varnothing$ & $\varnothing$ \\
\hline 12. & \multicolumn{3}{|c|}{ Discours direct + incise avec verbe dicendi +Discours direct(DD+iVD+DD) } \\
\hline 012BR & $\begin{array}{l}\text { [coelho falando para a lebre] - já sei } \\
\text { o que posso/fazer- disse o coelho } \\
\text { - vou apostaruma/corrida com } \\
\text { você. } \\
\text { [le lapin parlant au lièvre - je sais } \\
\text { qu je pew le faire-dit le lapin-je } \\
\text { parie une course avec toi. }\end{array}$ & $\varnothing$ & $\mathrm{C}_{\mathrm{C}} \mathrm{C}$ \\
\hline 13. & \multicolumn{3}{|c|}{ Discours direct du narrateur (DDn) } \\
\hline$\varnothing$ & $\varnothing$ & 024FR & $\begin{array}{l}\text { [narrateur en parlent au lecteur] Et } \\
\text { maintenant, / quand c'est quill y a des loups } \\
\text { garous dans / le coin, on fait attention. }\end{array}$ \\
\hline 14. & \multicolumn{3}{|c|}{ Discours indirect libre sans verbe introducteur (DIL) } \\
\hline$\varnothing$ & $\varnothing$ & $005 \mathrm{FR}$ & $\begin{array}{l}\text { [et soudain quand il [Thomme préhistorique] } \\
\text { a entendu un bruit! splatche!] C'était un / truc } \\
\text { bizarre. }\end{array}$ \\
\hline
\end{tabular}

